\title{
Identification and analysis of microRNAs in Botryococcus braunii using high-throughput sequencing
}

\author{
Xiang-Yuan Deng*, Xiao-Li Hu, Da Li, Ling Wang, Jie Cheng, Kun Gao \\ College of Biotechnology, Jiangsu University of Science and Technology, Zhenjiang 212003, PR China
}

\begin{abstract}
MicroRNAs (miRNAs) play important regulatory roles in the growth and development of organisms. The colonial green microalga Botryococcus braunii is an oil-rich algae and little is known about its miRNAs and their target genes. Here we constructed and sequenced a small RNA library for $B$. braunii using the HiSeq 2000 deep sequencing method. In total, we identified 42 known miRNA families and 14 novel miRNAs from $B$. braunii via sequence alignment and secondary structure prediction. Quantitative real-time PCR analysis indicated that the majority of miRNAs were effective and credible. Gene ontology analysis showed that most of the targets of miRNAs were classified as being involved in metabolic and cellular processes, gene expression regulation and stress/defense functions. Our findings provide the first large-scale identification and characterization of $B$. braunii miRNAs and their potential target genes. This study could lead to further identification of $B$. braunii miRNAs and enhance our understanding of their regulatory mechanisms in diverse biological and metabolic processes.
\end{abstract}

KEY WORDS: Botryococcus braunii $\cdot$ MicroRNAs $\cdot$ High-throughput sequencing $\cdot$ Target genes

\section{INTRODUCTION}

MicroRNAs (miRNAs) are small non-proteincoding RNAs with a length of approximately 22 nucleotides (nt), which have emerged as important regulators of genes at both transcriptional and posttranscriptional levels through translational repression, mRNA degradation and chromatin modification (Czech \& Hannon 2011, Prakash et al. 2016). To date, numerous miRNAs have been identified from various plant species (Lu et al. 2008, Pantaleo et al. 2010, Jin et al. 2013, Ferdous et al. 2015, Pantaleo et al. 2016), and a few reports have been published recently to describe the structure and function of miRNA from algae, such as Chlamydomonas reinhardtii (Zhao et al. 2007, Valli et al. 2016), Thalassiosira pseudonana (Norden-Krichmar et al. 2011), Phaeodactylum tricornutum (Huang et al. 2011), Porphyra yezoensis (Liang et al. 2010) and Porphyridium purpureum (Gao et al. 2016).

${ }^{*}$ Corresponding author: dengxy2009@126.com
Microalgae have the potential to generate significant quantities of biomass and oil suitable for conversion to biodiesel. The colonial green microalga Botryococcus braunii has a remarkable ability to produce high levels of liquid hydrocarbons (Banerjee et al. 2002, Metzger \& Largeau 2005). In previous studies, $B$. braunii was classified into 3 races (A, B and L) based on hydrocarbon structures (Kawachi et al. 2012, Yoshimura et al. 2013), and the genome and transcriptome of $B$. braunii were sequenced and analyzed (Weiss et al. 2011, Ioki et al. 2012a,b). To date, no systematic studies have been conducted on small RNA in this microalga. Thus, the aim of our study was to isolate miRNAs from $B$. braunii and (1) to establish a small RNA library of $B$. braunii, (2) to obtain sequences and information of miRNAs in $B$. braunii, and (3) to identify novel miRNAs in $B$. braunii using bioinformatics methods. The results will help us to identify the miRNA-based regulatory system of this microalga.

() The authors 2017. Open Access under Creative Commons by Attribution Licence. Use, distribution and reproduction are unrestricted. Authors and original publication must be credited. 


\section{MATERIALS AND METHODS}

\author{
Strains and culture conditions
}

Botryococcus braunii was obtained from the Freshwater Algae Culture Collection, Institute of Hydrobiology, Chinese Academy of Sciences, and propagated photoautotrophically in 11 Erlenmeyer flasks containing $400 \mathrm{ml}$ of modified Chu-13 medium (Largeau et al. 1980). The flasks were placed in a $25 \pm 1^{\circ} \mathrm{C}$ illuminated incubator (Jiangnan Instrument Factory) for $14 \mathrm{~d}$ under a 14:10 h light:dark photoperiod and a light density of $50 \pm 5 \mu \mathrm{E} \mathrm{m} \mathrm{m}^{-2} \mathrm{~s}^{-1}$. The microalgal cells were then collected and cleaned with sterilized water. After being dried with hygroscopic filter paper, the samples were immediately frozen in liquid nitrogen and stored at $-80^{\circ} \mathrm{C}$ before use.

\section{Construction and sequencing of a small RNA library}

Total RNA used for small RNA library construction was extracted from frozen $B$. braunii cells using Trizol (Invitrogen, Life Technologies) according to the manufacturer's instructions. Briefly, the frozen cells were re-suspended in $3 \mathrm{ml}$ of Trizol reagent. Total RNA was then extracted and purified with the standard Trizol/RNeasy column procedure. Following purification, total RNAs were digested with DNase and quantified by spectrophotometry. Total RNAs were then separated by size fractionation on denaturing $15 \%$ polyacrylamide gels. Fragments of 18 to 28 nt were gel-purified and then ligated to a 5'-adaptor and a 3'-adaptor. After reverse transcription, a 12cycle PCR reaction was performed, and the products were sequenced by the Beijing Genomics Institute (BGI) using the HiSeq 2000 deep sequencing method.

\section{Small RNA analysis and miRNA identification}

For analysis of small RNAs in B. braunii, unique small RNAs were compared and aligned with the sequences of non-coding RNAs (rRNA, tRNA, snRNA, snoRNA) available in Rfam (www.sanger.ac. uk/software/Rfam) (Griffiths-Jones et al. 2005) and in the GenBank noncoding RNA database (www.ncbi. nlm.nih.gov/) using blastn with an e-value of 0.01 as the cutoff. In addition, all sequences were searched for conserved miRNAs of $B$. braunii using the plant mature miRNAs from miRBase (release 19.0) allow- ing for 2 mismatches and 3 gaps (Griffiths-Jones et al. 2008).

\section{Prediction of novel miRNAs}

Fold-back structures in miRNA precursors can be used to predict novel miRNAs (Allen et al. 2005). In this study, the Mireap program, developed by the BGI, was used to predict the novel miRNAs in $B$. braunii. The adopted strategy was as follows: (1) candidate miRNA sites were screened out from breakpoints defined by mapping of the small RNAs, (2) a minimal stringent criterion was used to select miRNA candidates and (3) the RNA secondary structure was checked with the Mfold program (Zuker 2003).

\section{Prediction of miRNA targets}

The miRanda program (www.microrna.org/) was used to detect potential target sites for the $B$. braunii candidate miRNA sequences. The parameters employed were as follows: match score $S \geq 90$, target duplex free energy $\Delta G \leq-20 \mathrm{kcal} \mathrm{mol}^{-1}$ and scaling parameter $=2$. The miRNA-target duplexes were then checked manually according to rules suggested by John et al. (2004) and Huang et al. (2011).

\section{Quantitative real-time PCR analysis}

Ten miRNAs were randomly selected for quantitative real-time PCR (qRT-PCR) analysis. TaqMan miRNA assays were designed and ordered from Applied Biosystems. Each TaqMan miRNA assay includes a specific RT primer, forward and reverse primers and the TaqMan probe. Specific primers were designed according to the method of Chen et al. (2005) and synthesized by Sangon Biotechnology (Shanghai, China). Primers used in this study are listed in Table 1. qRT-PCR was performed according to the manufacturer's protocol and run on an ABI 7300 machine (Applied Biosystems) with thermal cycling parameters at $95^{\circ} \mathrm{C}$ for $10 \mathrm{~min}$ followed by 40 cycles of $95^{\circ} \mathrm{C}$ for $30 \mathrm{~s}, 60^{\circ} \mathrm{C}$ for $1 \mathrm{~min}$ according to the manufacturer's protocol. qRT-PCR reactions were run in triplicate with 2 biological replicates. The U6 snRNA was selected as a reference gene for normalization. A relative quantitative method $\left(\Delta \Delta C_{t}\right)$ was used to evaluate relative expression levels of different miRNAs in B. braunii. 
Table 1. Primers used for quantitative real-time PCR of miRNAs from Botryococcus braunii

\begin{tabular}{|c|c|c|}
\hline MicroRNA & Primer & Primer sequence $\left(5^{\prime}-3^{\prime}\right)$ \\
\hline \multirow[t]{2}{*}{ miR1 } & RT & GTCGTATCCAGTGCAGGGTCCGAGGTATTCGCACTGGATACGACCCCCCCTCA \\
\hline & Forward & ATGGTTCGTGGGAGGGGTGAGAGGGGGTGAGG \\
\hline \multirow[t]{2}{*}{ miR3 } & $\mathrm{RT}$ & GTCGTATCCAGTGCAGGGTCCGAGGTATTCGCACTGGATACGACCCCCCCTCT \\
\hline & Forward & ATGGTTCGTGGGGAGGGGAAGAGGGGAAGAGG \\
\hline \multirow{2}{*}{ miR4 } & $\mathrm{RT}$ & GTCGTATCCAGTGCAGGGTCCGAGGTATTCGCACTGGATACGACCCCTCTACC \\
\hline & Forward & ATGGTTCGTGGGGAGGGGTAGAGGGGTAGAGG \\
\hline \multirow[t]{2}{*}{$\operatorname{miR6}$} & RT & GTCGTATCCAGTGCAGGGTCCGAGGTATTCGCACTGGATACGACCAACTACCA \\
\hline & Forward & ATGGTTCGTGGGGTAGTTGGTAGTTGGTAGTT \\
\hline \multirow[t]{2}{*}{$\operatorname{miR7}$} & $\mathrm{RT}$ & GTCGTATCCAGTGCAGGGTCCGAGGTATTCGCACTGGATACGACCTCCCCCCT \\
\hline & Forward & ATGGTTCGTGGGGGGAGAGGGGAGAGGGGGGA \\
\hline \multirow[t]{2}{*}{ miR8 } & RT & GTCGTATCCAGTGCAGGGTCCGAGGTATTCGCACTGGATACGACCCCCCTCTC \\
\hline & Forward & ATGGTTCGTGGGAGGGGGAGAGGGGGAGAGG \\
\hline \multirow[t]{2}{*}{$\operatorname{miR9}$} & $\mathrm{RT}$ & GTCGTATCCAGTGCAGGGTCCGAGGTATTCGCACTGGATACGACCCCCСTCTC \\
\hline & Forward & ATGGTTCGTGGGAGGGGGAGAGGGGAGAGG \\
\hline \multirow[t]{2}{*}{ miR10 } & $\mathrm{RT}$ & GTCGTATCCAGTGCAGGGTCCGAGGTATTCGCACTGGATACGACCCCCCСCTC \\
\hline & Forward & ATGGTTCGTGGGGGGAGAGGGGGGAGAGGG \\
\hline \multirow[t]{2}{*}{ miR12 } & RT & GTCGTATCCAGTGCAGGGTCCGAGGTATTCGCACTGGATACGACCCCCCTCTC \\
\hline & Forward & ATGGTTCGTGGGAGGGGGAGAGGGGGAGAGG \\
\hline \multirow[t]{2}{*}{$\operatorname{miR} 13$} & RT & GTCGTATCCAGTGCAGGGTCCGAGGTATTCGCACTGGATACGACCTCCCCATC \\
\hline & Forward & ATGGTTCGTGGGGGAAGGAGGGGGAGATGG \\
\hline \multirow[t]{3}{*}{ U6 } & $\mathrm{RT}$ & GTCGTATCCAGTGCAGGGTCCGAGGTATTCGCACTGGATACGACCAAATATGGAAC \\
\hline & Forward & CTCGCTTCGGCAGCACA \\
\hline & Reverse & AACGCTTCACGAATTTGCGT \\
\hline \multicolumn{2}{|c|}{ Universal reverse } & GCAGGGTCCGAGGTATTC \\
\hline
\end{tabular}

\section{RESULTS AND DISCUSSION}

\section{Sequencing and analysis of the small RNA library}

A small RNA library was established using Solexa technology to identify candidate miRNAs in Botryococcus braunii. In total, we obtained 10020143 sequence reads from the library. After filtering out low-quality tags, trimming adaptors and cleaning up shortages and contamination formed by adaptoradaptor ligation, we obtained 9786842 (98.08\%) clean reads, representing 2005344 unique sequences. Among the clean reads, $131905(1.35 \%)$ were similar to known miRNAs, and 6602059 $(67.46 \%)$ were unannotated small RNA, suggesting that small RNA in $B$. braunii has not been investigated extensively in previous studies. Thus, our study has potential to discover more miRNA genes. The rest of the sequences were other types of RNA, including tRNA, rRNA, snRNA, snoRNA and other non-coding RNAs. The numbers and proportions of different categories of small RNAs are shown in Table 2.
As an important feature, size profile has often been used to distinguish miRNA from other small RNAs in previous studies. Most mature miRNAs with known functions commonly have a length of 20-24 nt (Wei et al. 2009). In this study, the length distribution pattern of the reads was analyzed and is presented in Fig. 1, showing that the majority of small RNAs in $B$. braunii library were 21 and $20 \mathrm{nt}$ in size, accounting for 11.40 and $11.39 \%$ of the total reads, respectively (Fig. 1), followed by $19 \mathrm{nt}(10.59 \%), 22 \mathrm{nt}(10.17 \%), 23 \mathrm{nt}$ $(9.57 \%)$, and $24 \mathrm{nt}(8.87 \%)$. This distribution pattern

Table 2. Distribution of small RNAs among different categories in Botryococcus braunii

\begin{tabular}{|lcccr|}
\hline \multirow{2}{*}{ Category } & \multicolumn{2}{c}{ Unique RNAs } & \multicolumn{2}{c|}{ Total RNAs } \\
& Number & Percent & Number & Percent \\
\hline Total & 2005344 & 100.00 & 9786842 & 100.00 \\
exon_antisense & 121 & 0.01 & 440 & 0.00 \\
exon_sense & 364 & 0.02 & 947 & 0.01 \\
intron_antisense & 1217 & 0.06 & 9609 & 0.1 \\
intron_sense & 1203 & 0.06 & 12689 & 0.13 \\
miRNA & 14887 & 0.74 & 131905 & 1.35 \\
rRNA & 110727 & 5.52 & 1444330 & 14.76 \\
repeat & 53 & 0.00 & 89 & 0.00 \\
snRNA & 1381 & 0.07 & 27088 & 0.28 \\
snoRNA & 175 & 0.01 & 546 & 0.01 \\
tRNA & 40923 & 2.04 & 1557140 & 15.91 \\
Non-annotated sRNAs & 1834293 & 91.47 & 6602059 & 67.46 \\
& & & & \\
\hline
\end{tabular}




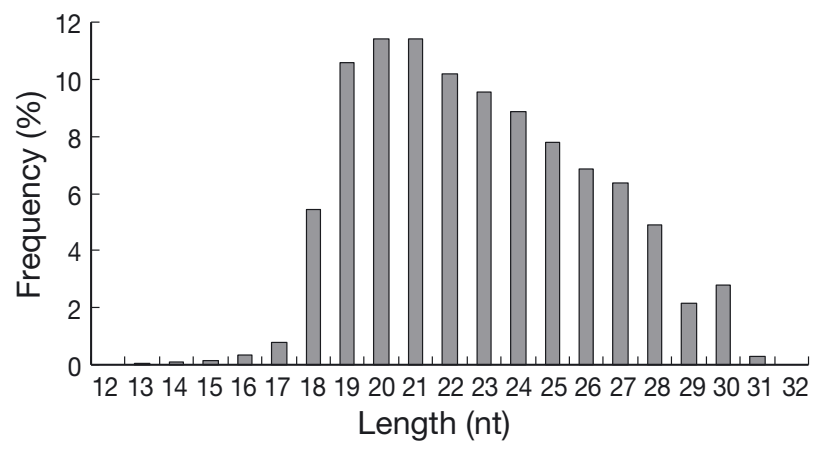

Fig. 1. Lengths of unique small RNA sequences in Botryococcus braunii. The occurrences of each unique sequence read were counted to reflect the relative expression level, and only small RNA sequences in the range of 12 to $32 \mathrm{nt}$ were considered

is highly consistent with the typical size of miRNA from Dicer digestion products and previous other algal small RNA sequencing using Solexa technology, such as Chlamydomonas reinhardtii (Zhao et al. 2007, Valli et al. 2016), Porphyra yezoensis (Liang et al. 2010), Phaeodactylum tricornutum (Huang et al. 2011) and Porphyridium purpureum (Gao et al. 2016).

\section{Identification of known miRNAs in B. braunii}

To identify the known miRNAs in $B$. braunii, the small RNA sequences were compared with the known plant miRNAs in miRBase 19.0 (www. mirbase.org/). Among the 9786842 sequences, 14887 unique sequences were orthologs of known miRNAs from other plant species previously deposited in miRBase (version 19.0). Allowing 1 or 2 mismatches between sequences, these miRNAs represented 42 known miRNA families (Table 3). Solexa sequencing technology has the ability to generate millions of small RNA sequences, which could provide a resource with information on the abundance of various miRNA families and even distinguish between different members of a given family (Ruan et al. 2009). Thus, as a powerful tool, Solexa sequencing technology is often used to estimate the expression profiles of miRNA genes. In this study, the frequencies of known miRNA families in the sequenced library varied from 6 (miR164a) to 41735 (miR408a-5p), indicating that expression varies significantly among different miRNA families. Among them, 13 miRNA families had more than 1000 sequence counts, suggesting these miRNAs were highly expressed in $B$. braunii.
Table 3. Putative miRNA families represented in the Botryococcus braunii miRNA pool

\begin{tabular}{|c|c|c|}
\hline $\begin{array}{l}\text { miRNA } \\
\text { family }\end{array}$ & Count & Sequence \\
\hline $\operatorname{miR} 157 a$ & 798 & TTGACAGAAGATAGAGAGCAC \\
\hline $\operatorname{miR} 6180$ & 1886 & AGGGGCTAGAAAGAGGGCAG \\
\hline miR6426a & 30629 & GTGGACGAAACGGAAGTGAGA \\
\hline miR167a & 313 & TGAAGCTGCCAGCATGATCTA \\
\hline $\operatorname{miR} 408 a-5 p$ & 41735 & CAGGGGAGCAGGGGAGCAG \\
\hline miR166a & 157 & TCGGACCAGGCTTCATTCCCC \\
\hline miR158a & 172 & TCCCAAATGTAGACAAAGCA \\
\hline miR5519 & 2399 & TGGCAGACGCTACGGACTTAA \\
\hline miR5742 & 492 & CCACATAAGGGTCGTTGTA \\
\hline $\operatorname{miR5076}$ & 609 & GAAATGGTGTAGCGGAGCAGGATT \\
\hline $\operatorname{miR} 5077$ & 162 & GAATCGCGTCGGGGTCACCA \\
\hline miR3948 & 34938 & GGGAGTAGGGAGTAGGGAGTAGGGAG \\
\hline miR535a & 48 & TGACAACGAGAGAGAGCACGC \\
\hline $\operatorname{miR} 6164 a$ & 442 & TCCAAAAGTGTAACGGAGG \\
\hline $\operatorname{miR} 1115-5 p$ & 710 & TGAGGTAAGAGCATTTGGTGG \\
\hline $\operatorname{miR} 844-5 p$ & 742 & AGTAGGACTAGGAGATGCT \\
\hline $\operatorname{miR} 394 b-3 p$ & 5953 & AGGTGGGGATGACGTCAAGT \\
\hline miR168a & 50 & TCGCTTGGTGCAGGTCGGGAC \\
\hline miR397a & 39 & TCATTGAGTGCAGCGTTGATGT \\
\hline miR169h & 831 & GGCGACATACTGGCTCATT \\
\hline $\operatorname{miR} 6478$ & 56 & GCCGCCTTAGCTCAGATGGTT \\
\hline miR161-5p.1 & 15 & TTGAAAGTGACTACATCGGGG \\
\hline miR165a & 18 & TCGGACCAGGCTTCATCCCCC \\
\hline miR854a & 1058 & GTTGAGGATGGGGGGAGGG \\
\hline miR5026 & 11 & ACTCATAAGATCGTGACACGT \\
\hline $\operatorname{miR6173}$ & 1374 & TAGCCGTAAACGATGGATAC \\
\hline miR390a & 17 & AAGCTCAGGAGGGATAGCGCC \\
\hline miR5501 & 6234 & ACTTGTGGCTAGGGGTGAA \\
\hline $\operatorname{miR} 5568 c-3 p$ & 101 & ACTACGAGTGTGGAACGGAGG \\
\hline miR894 & 23 & GTTTCACGTCGGGTTCACCA \\
\hline miR5656 & 73 & ACTTGAAGTAGACGATTTGGATT \\
\hline $\operatorname{miR} 173-5 p$ & 9 & TTCGCTTGCAGAGAGAAATCAC \\
\hline $\operatorname{miR} 841 b-5 p$ & 4843 & ACAGGCAGTGGAAACTGAA \\
\hline miR5503 & 1408 & TTCGGATTGTCAGAGGCACT \\
\hline miR164a & 6 & TGGAGAAGCAGGGCACGTGCA \\
\hline $\operatorname{miR} 4414 a-5 p$ & p 427 & AGCTGCTGACTGTATGGTTGT \\
\hline miR916 & 1088 & CAAGAGGTCGTCGGTTCGAATCC \\
\hline $\operatorname{miR} 2876-5 p$ & 366 & AATGGTGGCTGCGACTGTTTA \\
\hline $\operatorname{miR5673}$ & 5805 & GCGGAACTGGCGGAAGACAT \\
\hline $\operatorname{miR} 482-5 p$ & 51 & AGGGAGTGAAGGAGCGCCT \\
\hline miR6300 & 13 & GGTGGTTGTAGTATAGCGGT \\
\hline miR3633a-5p & p 846 & GGAATGGATGGTGTAGCG \\
\hline
\end{tabular}

miR408a-5p, miR3948, and miR6426a were the most frequently expressed miRNA, with 41735, 34938 , and 30629 copies, respectively. Moreover, 15 miRNA families had 100 to 1000 sequence counts, and others had fewer than 100 sequence counts. 
Table 4. Predicted novel miRNAs in Botryococcus braunii

\begin{tabular}{|cclccc|}
\hline Name & Count & Sequence & $\begin{array}{c}\text { Length } \\
\text { (nt) }\end{array}$ & $\begin{array}{c}\text { Precursor } \\
\text { length } \\
\text { (nt) }\end{array}$ & $\begin{array}{r}\text { Folding } \\
\text { energy } \\
\left(\mathrm{kcal} \mathrm{mol}^{-1}\right)\end{array}$ \\
\hline miR1 & 19 & AGGGGTGAGAGGGGGTGAGGGGG & 23 & 188 & -90.6 \\
miR2 & 19 & AGGGGTGAGAGGGGGTGAGGGGG & 23 & 284 & -148.2 \\
miR3 & 10 & GAGGGGAAGAGGGGAAGAGGGGG & 23 & 241 & -122.6 \\
miR4 & 14 & GAGGGGTAGAGGGGTAGAGG & 20 & 340 & -139.8 \\
miR5 & 9 & GGGAGAGGGGGGAGAGGGGGG & 21 & 278 & -144.5 \\
miR6 & 11 & GTAGTTGGTAGTTGGTAGTT & 20 & 148 & -53.7 \\
miR7 & 8 & GGGAGAGGGGAGAGGGGGGA & 20 & 207 & -72.0 \\
miR8 & 124 & AGGGGGAGAGGGGGAGAGGGG & 21 & 189 & -98.2 \\
miR9 & 10 & AGGGGGAGAGGGGAGAGGGG & 20 & 263 & -108.6 \\
miR10 & 18 & GGGAGAGGGGGGAGAGGGGGG & 21 & 224 & -98.0 \\
miR11 & 8 & GAGGAGGTGAGGAGGTGAGG & 20 & 311 & -125.0 \\
miR12 & 124 & AGGGGGAGAGGGGGAGAGGGG & 21 & 151 & -77.2 \\
miR13 & 1106 & GGAAGGAGGGGGAGATGGGGA & 21 & 88 & -23.4 \\
miR14 & 6 & TACCAGTTACCAGTTACCAG & 20 & 342 & -113.9 \\
& & & & & \\
\hline
\end{tabular}

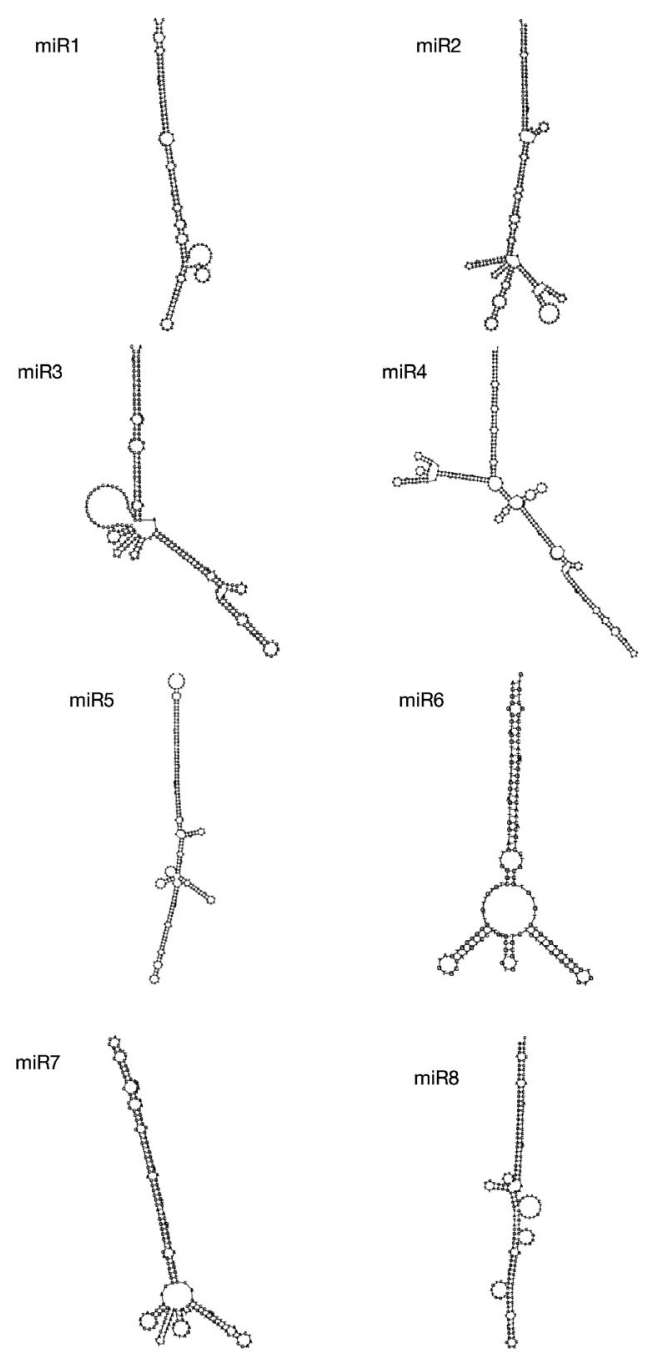

\section{Predicted novel miRNAs in $B$. braunii}

One of the important features that distinguishes miRNA from other small RNAs is the ability of miRNA flanking sequences to fold back into a hairpin structure (Ambros et al. 2003). Our search revealed that a total of 14 small RNAs met our criteria according to Allen et al. (2005) and were considered predicted novel miRNAs (Table 4). The lengths of these novel candidates
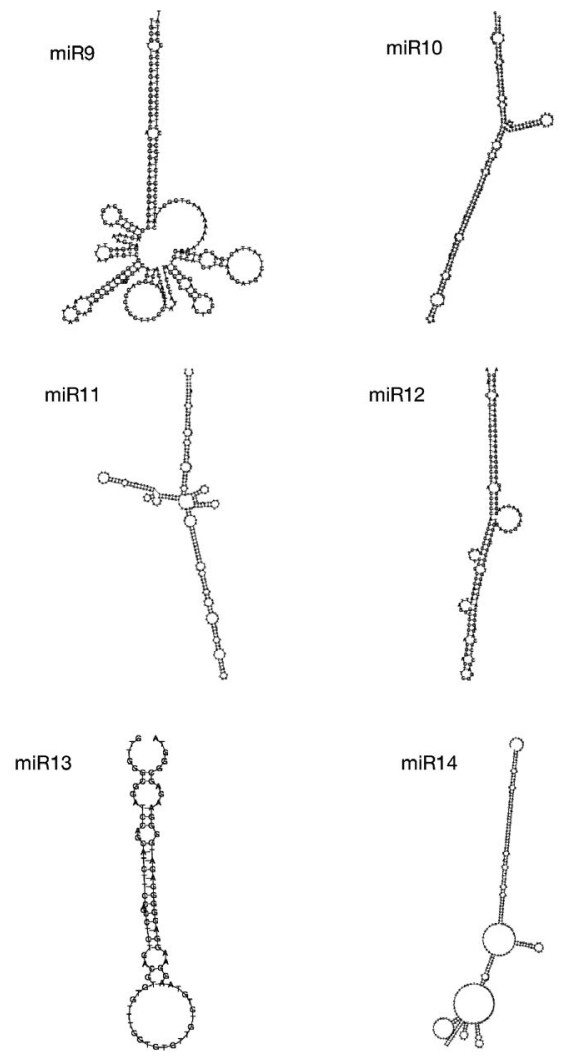

Fig. 2. Predicted hairpin structures of novel miRNAs in Botryococcus braunii 


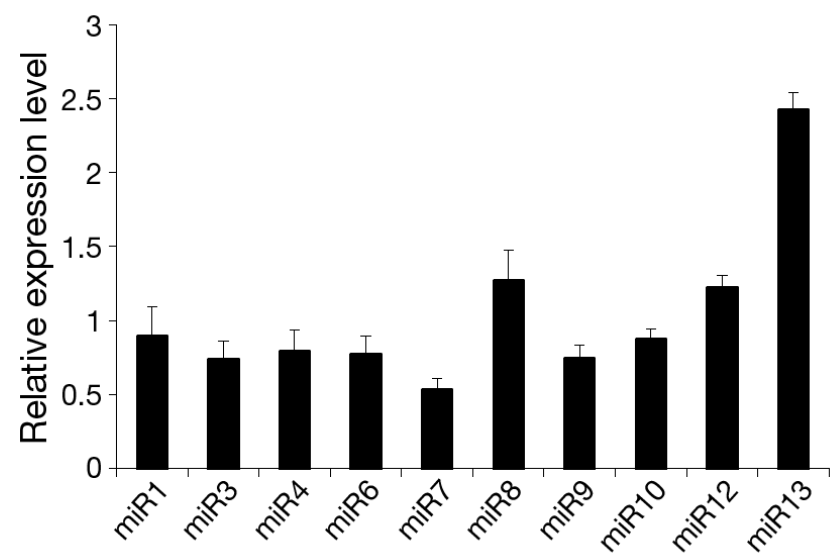

Fig. 3. Quantitative real-time PCR (qRT-PCR) validation and expression analysis of miRNAs in Botryococcus braunii. Data are mean \pm SD. qRT-PCR reactions were run in triplicate with 2 biological replicates

\section{Validation of novel miRNAs in B. braunii}

To validate the predicted novel miRNAs, $10 \mathrm{mi}-$ RNAs were randomly selected for qRT-PCR assays which were performed to examine whether the miRNAs were expressed in B. braunii. Fig. 3 shows that all 10 miRNAs were expressed in the samples of $B$. braunii, which was attributed to the conservation of miRNA families. The expression level of miR13 was relatively higher than that of the other 9 miRNAs, which was in agreement with the Solexa sequencing data (Table 4). These findings were also in line with those reported by Gao et al. (2016), who found that miRNAs are relatively conserved in different species. Moreover, these results suggest that a majority of the miRNAs identified in $B$. braunii are effective and credible. ranged from 20 to $23 \mathrm{nt}$, and precursors of these novel miRNAs had negative folding free energies ranging from -148.2 to $-23.4 \mathrm{kcal} \mathrm{mol}^{-1}$, with an average of about $-101.1 \mathrm{kcal} \mathrm{mol}^{-1}$ according to Mfold, which was much lower than terrestrial plant miRNA precursors $\left(-71.0 \mathrm{kcal} \mathrm{mol}^{-1}\right.$ in rice, -59.5 $\mathrm{kcal} \mathrm{mol}^{-1}$ in Arabidopsis and $-72.4 \mathrm{kcal} \mathrm{mol}^{-1}$ in wheat; Yao et al. 2007). The predicted hairpin structures (Fig. 2) for the precursors of these novel miRNAs required 88 to 342 nt, and a majority of the identified miRNA precursors $(71.4 \%)$ required 148 to $284 \mathrm{nt}$, which was more than what has been observed in other plants (Zhang et al. 2006). Moreover, the predicted novel miRNAs exhibited much lower expression levels. This finding was in line with the notion that the expression level of non-conserved miRNAs is lower than that of conserved miRNAs. Each novel miRNA family had only 1 member, and only the miR13 family had more than 1000 sequence reads. The majority of novel miRNA families had fewer than 20 reads. The low abundance of novel miRNAs might suggest a specific role for these miRNAs under various growth conditions, under biotic or abiotic stress, or during developmental stages (Zhao et al. 2010, Song et al. 2015).
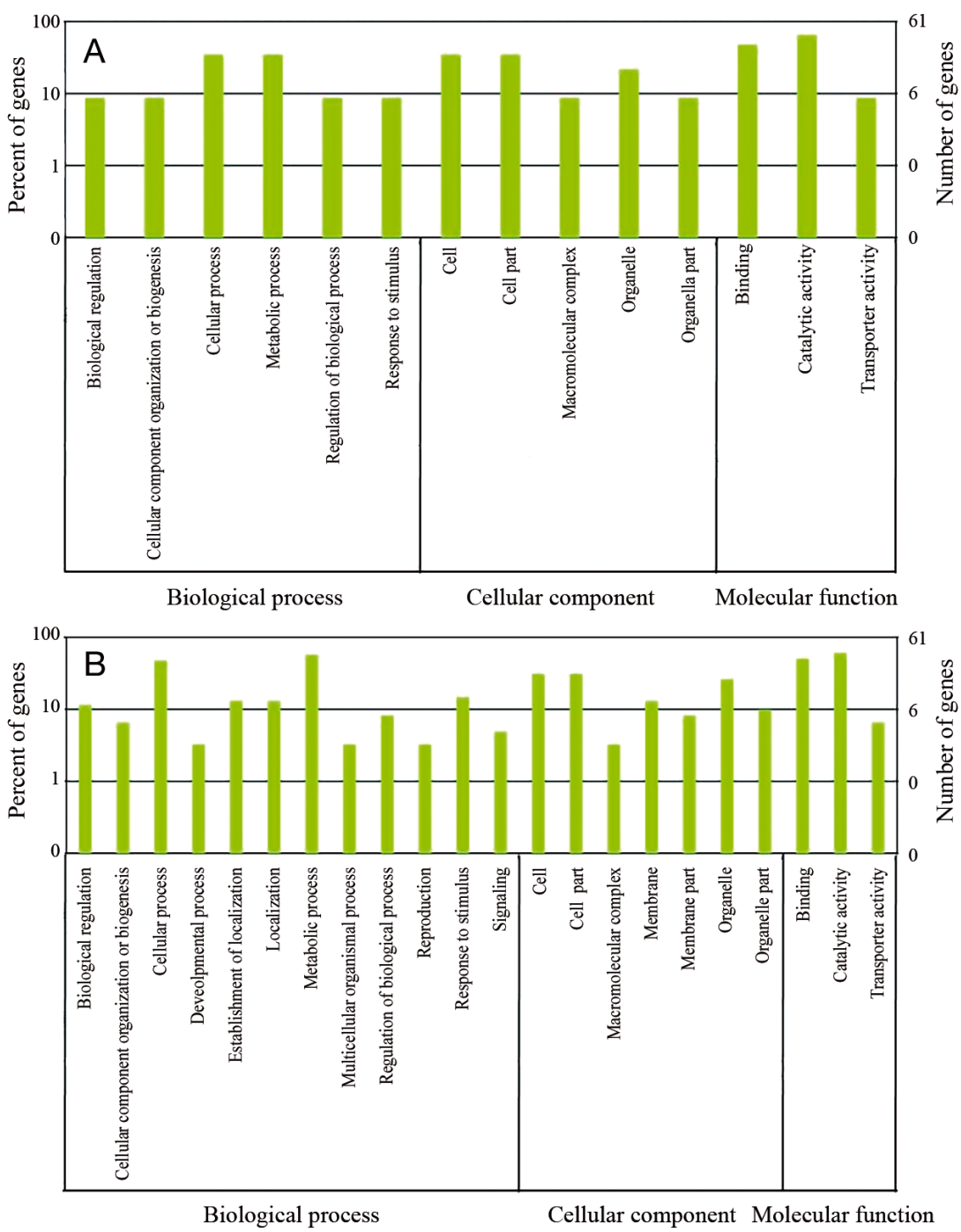

Fig. 4. Gene categories and distribution of (A) known miRNA targets and (B) novel miRNA targets in Botryococcus braunii 


\section{Prediction of miRNA targets in $B$. braunii}

In plants, miRNAs are involved in regulating a variety of biological processes, such as development, signal transduction, protein degradation, response to environmental stress and pathogen invasion (Lu et al. 2008). Their target sites have been shown to be primarily located in the coding regions. As expected, most miRNA target sites in B. braunii were located in the coding regions, and the putative target genes appear to be involved in a wide variety of biological processes (Fig. 4). Of these predicted targets, most were classified as being involved in metabolic and cellular processes, gene expression regulation, and stress/defense functions. Many metabolism networks have also been found, including lipid metabolism, amino acid metabolism, carbohydrate metabolism, energy metabolism, and nitrogen metabolism (Chi et al. 2011).

\section{CONCLUSIONS}

This is the first report of genome-wide identification of miRNAs in Botryococcus braunii using a highthroughput Solexa sequencing strategy. We have identified 42 known miRNA families and 14 novel miRNAs from $B$. braunii, suggesting that a significant number of novel microRNAs remain to be discovered and characterized. Additionally, we also predicted putative targets for these miRNAs. These results may help to improve our understanding of regulatory mechanisms of miRNAs in biological and metabolic processes of $B$. braunii.

Acknowledgements. This study was supported by the Jiangsu Provincial Natural Science Foundation of China (No. BK2011493) and China Postdoctoral Science Foundation funded project (No. 2013M531370, 2014T70532).

\section{LITERATURE CITED}

Allen E, Xie Z, Gustafson AM, Carrington JC (2005) microRNA-directed phasing during trans-acting siRNA biogenesis in plants. Cell 121:207-221

Ambros V, Bartel B, Bartel DP, Burge CB and others (2003) A uniform system for microRNA annotation. RNA 9: 277-279

Banerjee A, Sharma R, Chisti Y, Banerjee UC (2002) Botryococcus braunii: a renewable source of hydrocarbons and other chemicals. Crit Rev Biotechnol 22:245-279

Chen C, Ridzon DA, Broomer AJ, Zhou Z and others (2005) Real-time quantification of microRNAs by stem-loop RTPCR. Nucleic Acids Res 33:e179

* Chi X, Yang Q, Chen X, Wang J and others (2011) Identifi- cation and characterization of microRNAs from peanut (Arachis hypogaea L.) by high-throughput sequencing. PLOS ONE 6:e27530

* Czech B, Hannon GJ (2011) Small RNA sorting: matchmaking for Argonautes. Nat Rev Genet 12:19-31

Ferdous J, Hussain SS, Shi BJ (2015) Role of microRNAs in plant drought tolerance. Plant Biotechnol J 13:293-305

*Gao F, Nan F, Feng J, Lv J, Liu Q, Xie S (2016) Identification of conserved and novel microRNAs in Porphyridium purpureum via deep sequencing and bioinformatics. BMC Genomics 17:612

*Griffiths-Jones S, Moxon S, Marshall M, Khanna A, Eddy SR, Bateman A (2005) Rfam: annotating non-coding RNAs in complete genomes. Nucleic Acids Res 33 (Suppl 1):D121-D124

Griffiths-Jones S, Saini HK, van Dongen S, Enright AJ (2008) miRBase: tools for microRNA genomics. Nucleic Acids Res 36(Suppl 1):D154-D158

Huang A, He L, Wang G (2011) Identification and characterization of microRNAs from Phaeodactylum tricornutum by high-throughput sequencing and bioinformatics analysis. BMC Genomics 12:337

Ioki M, Baba M, Nakajima N, Shiraiwa Y, Watanabe MM (2012a) Transcriptome analysis of an oil-rich race B strain of Botryococcus braunii (BOT-22) by de novo assembly of pyrosequencing cDNA reads. Bioresour Technol 109: 292-296

Ioki M, Baba M, Nakajima N, Shiraiwa Y, Watanabe MM (2012b) Transcriptome analysis of an oil-rich race $B$ strain of Botryococcus braunii (BOT-70) by de novo assembly of 5 -end sequences of full-length cDNA clones. Bioresour Technol 109:277-281

Jin D, Wang Y, Zhao Y, Chen M (2013) MicroRNAs and their cross-talks in plant development. J Genet Genomics 40: 161-170

John B, Enright AJ, Aravin A, Tuschl T, Sander C, Marks DS (2004) Human microRNA targets. PLOS Biol 2:e363

Kawachi M, Tanoi T, Demura M, Kaya K, Watanabe MM (2012) Relationship between hydrocarbons and molecular phylogeny of Botryococcus braunii. Algal Res 1: 114-119

* Largeau C, Casadevall E, Berkaloff C, Dhamelincourt P (1980) Sites of accumulation and composition of hydrocarbons in Botryococcus braunii. Phytochemistry 19: 1043-1051

* Liang C, Zhang X, Zou J, Xu D, Su F, Ye N (2010) Identification of miRNA from Porphyra yezoensis by highthroughput sequencing and bioinformatics analysis. PLOS ONE 5:e10698

* Lu YD, Gan QH, Chi XY, Qin S (2008) Identification and characterization of microRNAs and their targets in grapevine (Vitis vinifera). Agric Sci China 7:929-943

*Metzger P, Largeau C (2005) Botryococcus braunii: a rich source for hydrocarbons and related ether lipids. Appl Microbiol Biotechnol 66:486-496

*Norden-Krichmar TM, Allen AE, Gaasterland T, Hildebrand M (2011) Characterization of the small RNA transcriptome of the diatom, Thalassiosira pseudonana. PLOS ONE 6:e22870

*Pantaleo V, Saldarelli P, Miozzi L, Giampetruzzi A and others (2010) Deep sequencing analysis of viral short RNAs from an infected Pinot Noir grapevine. Virology 408: 49-56

Pantaleo V, Vitali M, Boccacci P, Miozzi L and others (2016) Novel functional microRNAs from virus-free and in- 
fected Vitis vinifera plants under water stress. Sci Rep 6: 20167

Prakash P, Rajakani R, Gupta V (2016) Transcriptome-wide identification of Rauvolfia serpentina microRNAs and prediction of their potential targets. Comput Biol Chem 61:62-74

Ruan MB, Zhao YT, Meng ZH, Wang XJ, Yang WC (2009) Conserved miRNA analysis in Gossypium hirsutum through small RNA sequencing. Genomics 94:263-268

Song A, Wang L, Chen S, Jiang J, Guan Z, Li P, Chen F (2015) Identification of nitrogen starvation-responsive microRNAs in Chrysanthemum nankingense. Plant Physiol Biochem 91:41-48

Valli AA, Santos BACM, Hnatova S, Bassett AR, Molnar A, Chung BY, Baulcombe DC (2016) Most microRNAs in the single-cell alga Chlamydomonas reinhardtii are produced by Dicer-like 3-mediated cleavage of introns and untranslated regions of coding RNAs. Genome Res 26: 519-529

Wei B, Cai T, Zhang R, Li A and others (2009) Novel microRNAs uncovered by deep sequencing of small RNA transcriptomes in bread wheat (Triticum aestivum L.) and Brachypodium distachyon (L.) Beauv. Funct Integr Genomics 9:499-511

Editorial responsibility: Kedong Yin, Brisbane, Queensland, Australia
Weiss TL, Johnston JS, Fujisawa K, Okada S, Devarenne TP (2011) Genome size and phylogenetic analysis of the A and L races of Botryococcus braunii. J Appl Phycol 23: 833-839

Y Yao Y, Guo G, Ni Z, Sunkar R, Du J, Zhu JK, Sun Q (2007) Cloning and characterization of microRNAs from wheat (Triticum aestivum L.). Genome Biol 8:R96

Y Yoshimura T, Okada S, Honda M (2013) Culture of the hydrocarbon producing microalga Botryococcus braunii strain Showa: optimal $\mathrm{CO}_{2}$, salinity, temperature, and irradiance conditions. Bioresour Technol 133:232-239

* Zhang B, Pan X, Cannon CH, Cobb GP, Anderson TA (2006) Conservation and divergence of plant microRNA genes. Plant J 46:243-259

Zhao CZ, Xia H, Frazier TP, Yao YY and others (2010) Deep sequencing identifies novel and conserved microRNAs in peanuts (Arachis hypogaea L.). BMC Plant Biol 10:3

Zhao T, Li G, Mi S, Li S, Hannon GJ, Wang XJ, Qi Y (2007) A complex system of small RNAs in the unicellular green alga Chlamydomonas reinhardtii. Genes Dev 21: 1190-1203

* Zuker M (2003) Mfold web server for nucleic acid folding and hybridization prediction. Nucleic Acids Res 31: 3406-3415

Submitted: March 24, 2016; Accepted: January 12, 2017

Proofs received from author(s): February 10, 2017 